AperTO - Archivio Istituzionale Open Access dell'Università di Torino

Improving the preclinical models for the study of chemotherapy-induced cardiotoxicity: a Position Paper of the Italian Working Group on Drug Cardiotoxicity and Cardioprotection

This is a pre print version of the following article:

Original Citation:

Availability:

This version is available http://hdl.handle.net/2318/1522923

since 2016-06-03T14:09:18Z

Published version:

DOI:10.1007/s10741-015-9497-4

Terms of use:

Open Access

Anyone can freely access the full text of works made available as "Open Access". Works made available under a Creative Commons license can be used according to the terms and conditions of said license. Use of all other works requires consent of the right holder (author or publisher) if not exempted from copyright protection by the applicable law. 


\section{Improving the preclinical models for the study of chemotherapy-induced cardiotoxicity: a Position Paper of the Italian Working Group on Drug Cardiotoxicity and Cardioprotection}

Rosalinda Madonna ${ }^{1,2,3} \bullet$ Christian Cadeddu 4 Martino Deidda ${ }^{4} \bullet$ Donato Mele $^{5} \bullet$ Ines Monte ${ }^{6}$ Giuseppina

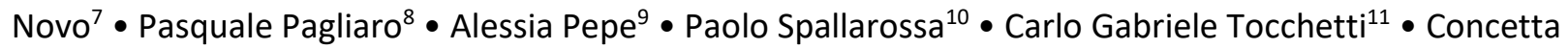
Zito $^{12} \cdot$ Giuseppe Mercuro ${ }^{4}$

1 Center of Excellence on Aging, Institute of Cardiology, "G. d'Annunzio" University - Chieti, Chieti, Italy 2 Texas Heart Institute, Houston, TX, USA

3 Cardiology Division, Department of Internal Medicine, University of Texas Medical School, Houston, TX, USA

4 Department of Medical Sciences "Mario Aresu", University of Cagliari, Cagliari, Italy

5 Cardiology Unit, University Hospital of Ferrara, Ferrara, Italy

6 Department of General Surgery and Medical-Surgery Specialties, University of Catania, Catania, Italy

7 Chair and Division of Cardiology, University of Palermo, Palermo, Italy

8 Department of Clinical and Biological Sciences, University of Turin, Orbassano, Italy

9 U.O.C. Magnetic Resonance Imaging, Fondazione G. Monasterio C.N.R., Pisa, Italy

10 Clinic of Cardiovascular Diseases, IRCCS San Martino IST, Genoa, Italy

11 Division of Internal Medicine, Department of Translational Medical Sciences, Federico II University, Naples, Italy

12 U.O.C. Cardiology Intensive Unit, A.O.U. Policlinico "G. Martino", University of Messina, Messina, Italy

Correspondence: Rosalinda Madonna rmadonna@unich.it; Giuseppe Mercuromercuro@medicina.unica.it

\section{Abstract}

Although treatment for heart failure induced by cancer therapy has improved in recent years, the prevalence of cardiomyopathy due to antineoplastic therapy remains significant worldwide. In addition to traditional mediators of myocardial damage, such as reactive oxygen species, new pathways and target cells should be considered responsible for the impairment of cardiac function during anticancer treatment. Accordingly, there is a need to develop novel therapeutic strategies to protect the heart from pharmacologic injury, and improve clinical outcomes in cancer patients. The development of novel protective therapies requires testing putative therapeutic strategies in appropriate animal models of chemotherapyinduced cardiomyopathy. This Position Paper of the Working Group on Drug Cardiotoxicity and Cardioprotection of the Italian Society of Cardiology aims to: (1) define the distinctive etiopatogenetic features of cardiac toxicity induced by cancer therapy in humans, which include new aspects of mitochondrial function and oxidative stress, neuregulin-1 modulation through the ErbB receptor family, angiogenesis inhibition, and cardiac stem cell depletion and/or dysfunction; (2) review the new, more promising therapeutic strategies for cardioprotection, aimed to increase the survival of patients with severe antineoplastic-induced cardiotoxicity; (3) recommend the distinctive pathological features of cardiotoxicity induced by cancer therapy in humans that should be present in animal models used to identify or to test new cardioprotective therapies.

Keywords Cancer therapy-induced cardiac injury Preclinical models Cardioprotection Mitochondria Neuregulin-1 Oxidative stress Statins Beta-blockers ACE inhibitors Cardiac stem cells 


\section{Abbreviations}

CHF Congestive heart failure

ROS Reactive oxygen species

NRG-1

Neuregulin-1

Top2

Topoisomerase II

$\mathrm{PI3}$

Phosphoinositide-3

AKT Protein kinase $B$

MEK/ERK Mitogen extracellular kinase

TK Tyrosine kinase

VEGFR Vascular endothelial growth factor receptor

PDGFR Platelet-derived growth factor receptor

CSCs Cardiac stem cells

CPCs Cardiac progenitor cells

ACE Angiotensin-converting enzyme

\section{Introduction}

Congestive heart failure (CHF) is the leading cause of disability in long-term survivors of cancer. The pathological consequences of $\mathrm{CHF}$ arise from the detrimental effects of antineoplastic drugs resulting in cardiomyocyte death, heart failure, arrhythmias, and death. The major concern is about cardiotoxicity associated with anthracyclines. Retrospective analyses from clinical trials in adults showed that the risk of $\mathrm{CHF}$ due to anthracyclines therapy is cumulative, dose related, and rises from $* 5 \%$ at doxorubicin dose of $400 \mathrm{mg} / \mathrm{m} 2$ to $16 \%$ at doses exceeding $500 \mathrm{mg} / \mathrm{m} 2$ [1]. This risk may be increased if we consider the cumulative effects of the drug over time. Using the Surveillance, Epidemiology, and End Results Medicare (SEER) database, it was found a cumulative $38 \%$ rate of CHF over 10 years in women aged 66 years and older who had received adjuvant anthracyclines [2]. This can be compared with a rate of $33 \%$ in women treated with regimens not containing an anthracycline and $29 \%$ in those with no adjuvant chemotherapy [2]. There is also concern about cardiotoxicity associated with the anti-HER- 2 monoclonal antibody trastuzumab. However, while anthracycline cardiotoxicity is largely irreversible, patients experiencing CHF when taking trastuzumab often recover [3]. Novel therapeutic interventions, which are capable of limiting cardiomyocyte death, are required to improve clinical outcomes in cancer patients. A variety of cardioprotective interventions has been investigated over the years in several small clinical trials, despite disappointing results. A through understanding of basic mechanisms of cancer therapy-induced cardiac toxicity is crucial for identifying new treatment strategies and novel targets for cardioprotection. Research to identify novel targets and cardioprotective therapies for cancer-induced cardiac injury usually requires preclinical testing in appropriate animal models. Although numerous animal models are available for use, there are inadequate standards for what clinical features of the cardiomyopathy due to antineoplastic therapy should be present in these models, and the presence or absence of the cancer-induced cardiotoxicity phenotype is often not documented. The aim of this Position Paper is first to define the 
distinctive etiopatogenetic features of cardiac toxicity induced by cancer therapy in humans; then to review the novel promising therapeutic strategies for cardioprotection; finally to recommend those distinctive pathological features of cardiac toxicity induced by cancer therapy in humans that should be present in an animal model being used to identify or to test novel cardioprotective therapies.

\section{Novel mechanisms of cardiotoxicity}

\section{Mitochondrial dysfunction and oxidative stress}

The antineoplastic activities of anthracyclines are due to several mechanisms of action, including the formation of topoisomerase II complex that impairs DNA replication as well as the induction of apoptotic cascades [4] and oxidative stress, which can occur via both enzymatic and nonenzymatic pathways [5]. Oxidative stress, due to generation of reactive oxygen species (ROS), is also the most widely accepted mechanism of anthracycline-induced cardiac toxicity $[6,7]$. Anthracyclines undergo redox cycling with generation of ROS such as superoxide anion $\left(\mathrm{O}^{-}\right)$and hydrogen peroxide $\left(\mathrm{H}_{2} \mathrm{O}_{2}\right)$, with consequent oxidative stress, energy depletion in cardiomyocytes, impaired mitochondrial function, cellular membrane damage, apoptosis, and cytotoxicity [8]. Recent data suggest that the cardiotoxicity induced by anthracyclines also occurs via the isozyme Top2b in cardiomyocytes. Anthracyclines are intercalating agent, capable of inserting between DNA base pairs and Top2b, leading to the formation of ternary Top2b-doxorubicin-DNA complex in the cardiomyocytes. This complex inhibits the re-ligation of the broken DNA strands, leading to DNA double-strand breaks and cell death [9]. In fact, after exposure to doxorubicin of cardiomyocytes harvested from conditional, cardiomyocytespecific Top $2 \mathrm{~b}$ knockout mouse, results marked reduction in DNA double-strand breaks, apoptotic nuclei, abnormalities in the p53 tumor suppressor gene, $b$-adrenergic signaling, and extrinsic and intrinsic apoptotic pathways as compared to wild-type mice [9]. With prolonged doxorubicin exposure, wild-type cardiomyocytes (but not Top2b KO cardiomyocytes) showed worse alterations in the expression of genes that regulate mitochondrial function, biogenesis, and oxidative phosphorylation [9], leading eventually to mitochondrial dysfunction and ROS generation [10]. Indeed, doxorubicin cardiotoxicity is blunted in mice knockout for the gene encoding the cardiac topoisomerase $2 \mathrm{~b}$ [10]. In mitochondria, anthracyclines may inhibit the electron transport chain process [11]. They have a high affinity for cardiolipin, which is involved in various phases of mitochondrial apoptotic process [11]. Nevertheless, ROS-dependent cardiolipin peroxidation may play a crucial role in the release of cytochrome C, which can also favor cardiolipin peroxidation, in a vicious cycle that ultimately leads to apoptosis [12]. Sildenafil, a phosphodiesterase- 5 inhibitor which has been proven to limit ischemia/reperfusion myocardial injury, can also protect against doxorubicin-induced myocardial toxicity [13, 14]. Cardioprotection by sildenafil against doxorubicin-induced myocardial injury was abolished by the nitric oxide synthase inhibitor L-NAME, which has been shown to inhibit mitochondrial ATP-sensitive potassium channels [15]. These results indicate that sildenafil's effect on nitric oxide pathway and on the opening of mitochondrial ATPsensitive potassium channels may limit doxorubicin-induced cardiotoxic effects.

\section{Inhibition of ErbB2}

ErbB2 is a member of the EGFR family. It is expressed in cardiomyocytes [16] and overexpressed in about $30 \%$ of breast cancers [17]. ErbB2 interacts with the other ErbBs, triggering signaling cascade that promotes tumor growth and survival [18]. The humanized anti-ErbB2 monoclonal antibody trastuzumab (herceptin) is effective in treating ErbB2? cancers, but unfortunately causes cardiotoxicity [19, 20], arising from impairment of contractility rather than loss of myocytes [21]. Neuregulin-1 (NRG1) is a small peptide 
and ligand receptor released from microvascular endothelial cells. In cardiomyocytes, NRG-1 acts via ErbB4/ErbB4 homodimers and ErbB4/ErbB2 heterodimers to stimulate protective pathways [22]. The ErbB2 pathway is needed for cell survival and function (PI3/AKT and MEK/ERK pathways) and is activated when the myocardium faces adverse hemodynamics or other stress, such as anthracyclines [23]. Activation of NRG1/ErbB pathway induces cell-cycle reentry of differentiated cardiomyocytes, with consequent cardiomyocyte proliferation and promotion of cardiac repair [24]. Trastuzumab cardiotoxicity is due to its binding to the extracellular domain of ErbB2 on cardiomyocytes, blocking myocardial ErbB2-ErbB4 cellprotective, growth promoting pathway [25-28]. Consistently, ErbB2 cardiac KO mice showed dilated cardiomyopathy, with enhanced myocytes death after anthracyclines [29]. Upon trastuzumab withdrawal, normal ErbB2 signaling is restored, and the decreased EF can return to normal, opposite to anthracyclines [30]. This is consistent with the enhanced cardiotoxicity occurring upon association of trastuzumab with anthracyclines: Trastuzumab amplifies the damage caused by anthracyclines. Once blocked the repair mechanisms because of ErbB2 blockade, the oxidative damage induced by anthracyclines proceeds without control [21]. Indeed, experimental studies have shown that neuregulin-1 modulates doxorubicin damage in rat cardiomyocytes $[4,16,27,31]$.

\section{Inhibition of angiogenesis}

Antiangiogenic drugs inhibit VEGF signaling and are associated with cardiovascular side effects $[32,33]$ such as arrhythmias, myocardial ischemia, and infarction, but in most cases hypertension and thromboembolism, LV dysfunction, and HF. Indeed, like cancer, the heart depends on adequate perfusion for its normal function [25, 28, 34-38], both relying on similar HIF-1 and VEGF pathways. In particular, the heart is sensitive to antiangiogenic therapy especially in the setting of stress such as hypertensionrelated pressure overload. Bevacizumab (avastin) is an antibody which binds circulating VEGF-A, currently approved for the treatment of advanced carcinoma of the lung, breast, colon, rectum [39]. It has been reported to induce left ventricle dysfunction ( $3 \%$ after prior chemotherapy; $1 \%$ with bevacizumab alone) [40]. Instead, sunitinib (sutent) and sorafenib (nevaxar) are small molecule tyrosine kinase inhibitor (TKI) with strong antiangiogenic activity, used in metastatic renal cancer and in imatinib-resistant gastrointestinal stromal tumors (GIST) [41, 42]. Interestingly, sunitinib and sorafenib are not very selective [34], since they target also other kinases, besides VEGF receptors. Sorafenib inhibits at least 15 kinases, including the PDGF receptor as well as VEGFRs and Raf-1/B-Raf, including KIT and FLT3 [25, 34, 35]. Sunitinib is reported to be more cardiotoxic, causing a decrease in left ventricle ejection fraction (LVEF) in up to $28 \%$ of patients [43-46], since it inhibits more than 30 other kinases, including c-kit, plateletderived growth factor receptor (PDGFR) alpha and beta, rearranged during transfection (RET), FMS-related tyrosine kinase 3 (FLT3), and colony-stimulating factor 1 receptor (CSF1R) [25, 34, 35, 47]. Seminal studies [48-52] have proven the importance of such pathways in cardiovascular homeostasis. The higher incidence of sunitinib cardiotoxicity is also explained by inhibition of off-target kinases, such as ribosomal 56 kinase (RSK), with consequent activation of the intrinsic apoptotic pathway, and $50 \mathrm{AMP}$-activated protein kinase (AMPK, important for the response to energy stress), with worsening of ATP depletion $[25,53]$. Therefore, LV dysfunction would occur due to myocyte dysfunction.

\section{Stem cells}

The adult heart has been recently shown to contain specific cardiac stem cells (CSCS) and cardiac progenitor cells (CPCs) that are cycling and can provide cell replacement for dying cardiomyocytes [54,55]. These cell populations may be sensitive to the interference of anthracyclines on DNA replicative machinery, resulting in the inhibition of CSC/CPC proliferation. This phenomenon may be operative in anthracycline-induced 
cardiomyopathy, so that repeated administration of the drug may affect the population of CSCs/CPCs that are activated in an attempt to repair the damaged myocardium. In fact, recent data have shown that exposure to doxorubicin over a period of 6 weeks led to an almost complete depletion of the CSC/ CPC pool within the myocardium, due to inhibition of CSC/CPC division in combination with the accumulation of oxidative DNA damage, growth arrest, cellular senescence, and apoptosis. This results in negative interference with the physiological turnover of cardiomyocytes and their regeneration in the presence of diffuse cell death [56]. Moreover, impaired high-energy phosphate metabolism and increased production of ROS might also affect negatively the function and survival of all cardiac compartments including CSCs/CPCs. All these negative effects can result in an impairment of myocyte replacement. Inhibition of the stem cell growth factor receptor c-kit is an additional mechanism by which stem cells may contribute to cardiotoxicity. Both sunitinib and sorafenib inhibit c-kit, which is expressed by precursors for hematopoietic stem cells, endothelial progenitor cells, and CPCs and is involved in the stem cell mobilization into the injury area [25]. Reduced c-kit kinase activity is proposed as additional mechanism of cardiotoxicity due to TK receptor inhibitors since it impairs the cardiac repair after injury [57].

\section{Novel cardioprotective strategies}

\section{Antioxidant therapies}

A variety of antioxidants has been studied for cardioprotection against anthracycline-induced cardiotoxicity in animal models and small human clinical trials, including probucol, vitamin $\mathrm{E}$, and $\mathrm{N}$-acetylcysteine, without benefits [58] (Table 1). The only evidence for prevention of anthracycline- induced cardiotoxicity has been shown for dexrazoxane, beta-blocker, angiotensin-converting-enzyme (ACE) inhibitors, and statins. Dexrazoxane is a pro-drug analog of the ethylenediaminetetracetic acid (EDTA), which acts as iron chelating agent, thereby preventing the formation of anthracycline-iron complexes and subsequent ROS formation. In addition to the antioxidative effects, dexrazoxane directly interferes with the formation of anthracycline-induced Top2a- and Top2b-DNA complexes, thereby reducing DNA damage [9]. A recent meta-analysis showed decreased risk of HF in cancer patients treated with dexrazoxane; however, the overall survival of these patients did not change [59]. Statins have been shown to exert cardioprotective effects against anthracycline-induced cardiotoxicity both in preclinical studies and in small human studies or retrospective observational studies. In vitro studies showed that lovastatin reduced doxorubicin-induced cardiomyocyte death and Top2b-mediated DNA damage [60]. Murine models of doxorubicin-induced cardiotoxicity showed attenuation of troponin I elevation and decreased cardiac fibrosis and left ventricular dysfunction after lovastatin cotreatment [61]. Retrospective observational studies showed reduction in hospitalization for HF in breast cancer patients co-treated with anthracyclines and statins [62]. In a small study of 40 patients treated with anthracyclines and randomized into statin group or control group, atorvastatin showed significant improvement of left ventricular ejection fraction as compared to control group [63]. In animal models of anthracycline-induced cardiotoxicity, aerobic exercise prior to and during anthracycline administration has been shown to exert cardioprotective effects in terms of decreased activation of pro-apoptotic cascade and increased proliferation of cardiomyocytes, essentially via reduction in ROS formation [64]. While exercise did not show beneficial effects in cancer patients treated with trastuzumab, there are small human studies showing reduction in all-cause mortality in anthracyclinetreated patients [65]. Further evidence will come from several registered large clinical trials evaluating the effect of exercise on anthracycline-induced cardiotoxicity (http:// www.clinicaltrials.gov/). Nutraceuticals, mostly phytochemicals derived from dietary or medicinal plants, such as soya bean, garlic, ginger, tea as well as propolis, honey, may have beneficial effects, mostly related to reduction in chemotherapy collateral effects, such as already suggested by epidemiologic studies [66]. Their ability to 
reduce cancer incidence in these studies is likely related to apoptosis and antioxidant properties. However, concerns exist about their real benefit in cancer patients which led to advise patients not to take them [67]. The concerns concentrate mainly on the interaction between the nutritional supplements and the chemotherapeutic agent used and its metabolization [68]. This is due to the fact that very often are not known the pharmacodynamics and pharmacokinetics of these substances in depth. Therefore, proper identification of molecular targets of nutraceuticals in vitro and preclinical studies is of primary importance to avoid offtarget effects and maximize their successful use in cancer patients.

\section{Angiotensin-converting-enzyme inhibitors}

Disruptions of the renin-angiotensin-aldosterone system (RAAS) are important in determining myocardial dysfunction induced by antineoplastic chemotherapies. Several small studies involving angiotensin II blockers administered during chemotherapies have investigated whether RAAS modulation could reduce antineoplastic-induced cardiomyopathy. For instance, ACE inhibitors have been shown to exert cardioprotective effects against anthracycline- induced cardiotoxicity both in animal and in small human studies [69], essentially via reduction in ROS formation [70]. Captopril and enalapril have been seen to reduce oxidative stress, downregulating the generation of ROS and preventing doxorubicin-induced cardiomyopathy [71]. ACE inhibitors may also reduce interstitial fibrosis [72] and may be protective via modulation of PPARb/c gene expression [73]. Yet candesartan, an angiotensin II type 1 receptor antagonist, reversed fibrosis and apoptosis and improved myocyte diameter/body weight ratio in a model of daunorubicin-induced cardiomyopathy [74]. The ACE inhibitor valsartan added to the CHOP chemotherapy (cyclophosphamide, doxorubicin, vincristine, and prednisolone) resulted protective improving echocardiographic and electrocardiographic parameters [75]. Finally, ACE inhibitors have been shown to exert cardioprotective effects against cardiotoxicity-induced trastuzumab via downregulation of NRG-/ErbB pathway [76]. Currently, there are several registered large clinical trials evaluating the effect of ACE inhibitors on trastuzumab- and anthracycline- induced cardiotoxicity (http://www.clinicaltrials. gov/).

\section{Beta-Adrenergic signaling blockers}

Although beta-blockers, such as carvedilol, nebivolol, alprenolol, have been recommended in preventing cardiac dysfunction during anthracycline treatment [77], clinical trials investigating the specific effects of this class of drug in HF patients with cancer are lacking or underway. In fact, most of the evidence comes from retrospective studies [78-82]. Compared to placebo, carvedilol seems to attenuate theLVEF decline and diastolic function impairment in patients treated with anthracyclines [81]. Similarly, a reduction in LVEF and an increase in LV volumes and brain natriuretic peptide (BNP) levels were shown in patients with breast cancer treated with placebo compared to those treated with nebivolol [79]. Additionally, a prognostic role was shown in patients with malignant hemopathies treated with anthracyclines, receiving placebo, or enalapril plus carvedilol. Patients treated with placebo experienced more frequently occurrence of death and HF. All patients had normal baseline EF and no variation of troponin levels. Echocardiographic data were also confirmed by cardiac magnetic resonance [82]. In other study examining the role of metoprolol or enalapril or placebo, the two drugs reduced the incidence of symptomatic HF, although the difference did not achieve significance [83]. Moreover, there was no difference in echocardiographic parameters of LV function among the 3 groups [83]. Possible mechanisms of beta-blockers-induced cardioprotection include downregulation of catecholamine stimulation with preservation of b-arrestin signaling pathway transactivation of ErbB1 (epidermal growth factorreceptor) [84] and prevention of myocardial calcium overload resulting in enhanced lusitropy [85]. Carvedilol also exhibits antioxidative effects [86]. More robust trials, such as prevention of left Ventricular dysfunction with Enalapril and caRvedilol in patients 
submitted to intensive ChemOtherapy for the treatment of Malignant hEmopathies (OVERCOME) [82], PRevention of cArdiac Dysfunction during Adjuvant breast cancer therapy (PRADA) [87], and MANTICORE101 breast (multidisciplinary approach to novel therapies in cardiology oncology research trial) [88], investigating the effects of combined treatment with betablockers and ACE inhibitors on anthracycline- and trastuzumab-induced cardiotoxicity, are undergoing.

\section{Stem cell therapy}

CSCs and CPCs offer novel and promising cardioprotective strategy for cancer therapy-induced cardiotoxicity, mainly through paracrine effects [56, 89-94]. Several signaling pathways including Akt [92] andNotch [91] are advocated to mediate the cardioprotective effects of CSCs and CPCs. It has been shown that transplantation of syngeneic CPCs via intramyocardial injection improves heart function in preclinical models of doxorubicin-induced cardiomyopathy [56]. Unfortunately, there are currently no registered clinical trials using CSCs or CPCs in cancer patients with cardiomyopathy (http://www.clinicaltrials.gov/). To date, preliminary results from preclinical studies are promising, although they remain inconclusive in terms of long-term effects. Extreme caution must be taken in the translation of these animal studies to humans. Nevertheless, these studies arise some possibility that myocardial biopsies may be obtained before antineoplastic drugs are given to cancer patients, and autologous CSCS or CPCs can be isolated and expanded from these myocardial samples and injected back into the cardiomyopathic heart of neoplastic patient. After transplantation, these cells can contribute to cardioprotection in the course of cancer therapyinduced cardiac injury, primarily via paracrine effects, i.e., secretion of growth factors. Evidence is accumulating that these released factors direct a number of restorative processes including myocardial protection, neovascularization, and reduction in cardiac fibrosis [91, 92].

\section{Preclinical models of chemotherapy-induced cardiotoxicity}

A variety of small and large animal models [95], as well as in vitro models [96], have been used to study the pathophysiology of cancer therapy-induced cardiomyopathy and pharmacological or nonpharmacological cardioprotective therapies. The limited translability of results from in vitro and animal models to human reflects the inability of any particular model to reproduce the pathophysiology of the disease in its complexity as in humans. Furthermore, results on toxic or therapeutic effects of antineoplastic drugs in various animal species are found extremely variable and not always similar to humans. Therefore, we can only partially compare precisely the dose and the toxic/ therapeutic effects of antineoplastic drugs between the animal model and the humans. Although small animals, such as murine and rat models, are low cost, readily available, easy to handle, results of studies evaluating cancer therapy-induced cardiomyopathy and its treatments in these models have offered only limited predictive clinical value. In rats, endovenous administration of anthracycline antibiotics causes morphologic lesions of the myocardium in a dose-related manner, with significant changes in the heart function in terms of intraventricular conduction defects and reduction in ejection fraction and mitochondria alterations in terms of inhibition of electron transfer, uncoupling of oxidative phosphorylation, and inhibition of calcium translocation [97]. However, these changes are more difficult to demonstrate than in larger animals. Large animal models, such as the rabbit and porcine models, have proven to be more predictive of cancer therapy-induced cardiomyopathy and its treatments in humans, although their predictive value is not absolute. The use of large mammalian models, however, is difficult to do, in part due to animal welfare restrictions and high costs of long-term monitoring of animals. In fact, because of chronic and delayed anthracycline cardiotoxicity [96], large animals require to be monitored for extended period. Even when large mammalian models are used, they most often do not take into consideration extreme age (i.e., children 
and very old people), co-morbidities, and comedications which a patient with cancer would most likely have. Taken together, lack of prediction and conflicting findings are related, in some instances, to speciesdependent sensitivity to cardiac injury - that does not necessarily reflect disease in humans - which in turn, is modulated by anatomical factors, metabolism, idiosyncratic toxicity reactions, immunological responses, as well as co-morbidities and their routine medications. Most experimental studies on cardioprotection are still undertaken in healthy juvenile animal models, although cancer therapy-induced cardiotoxicity in humans is a complex disorder that can be aggravated by or associated with cardiovascular risk factors and co-morbidities, including arterial hypertension, hyperlipidemia, diabetes, and aging. These risk factors induce fundamental alterations in cellular signaling cascades that affect the severity of antineoplastic druginduced cardiotoxicity and the responses to cardioprotective interventions [98]. Another important aspect to consider when testing the efficacy of a novel cardioprotective therapy is to ensure that the timing of the drug administration takes into account the clinical setting of the cancer therapy-induced cardiomyopathy. If early ventricular dysfunction in antineoplastic drug-treated patients is the intended clinical target, then it would be essential to demonstrate that the novel cardioprotective therapy can prevent early the left ventricular ejection fraction drop $\mathrm{C} 10 \%$ or even more sensitive parameters of left ventricular function at echocardiography or magnetic resonance imaging (MRI) when administered just prior or at the onset of anticancer treatment [99]. However, a decrease in left ventricular ejection fraction does not always predict early cardiac damage. In particular, markers of cardiomyocyte degeneration (such as serum levels of cardiac troponins) or elevated ventricular end-diastolic pressure or volume (such as serum levels of natriuretic peptides) or myocardial strain and strain rate as detected with Doppler echocardiography should be further evaluated in preclinical models $[95,96,100]$. Finally, the use of appropriate vehicle/sham control groups needs to be included in the study especially when pharmacological or stem cell-based therapeutic interventions are being considered [101, 102]. In conclusions, despite species-related or model-dependent differences, valuable insights into the molecular mechanisms of antineoplastic drug-induced cardiomyopathy can be deduced, but extrapolation from these animal findings to clinical relevance in humans should be made cautiously. Appropriate model application, respect and understanding of model limitations, and appropriate interpretation of the study results in light of model limitations should be taken into account. Therefore, continued development and characterization of suitable preclinical models are necessary in order to reproduce in animals antineoplastic drug-induced cardiomyopathy of humans and its treatment.

\section{The road ahead: toward clinical translation}

There are now a number of clinical and preclinical studies which have looked at cardioprotection by pharmacological agents, aerobic exercise, or advanced therapy (stem cell therapy) in various clinical scenarios of cancer therapy-induced cardiomyopathy. Most of the data regarding mechanisms of cardiotoxicity and cardioprotection have been obtained in animal models. Translatability of these results in the humans is always challenging; therefore, further studies are needed in order to assess the relevance of the pathways in human. With respect to pharmacological cardioprotection, both positive and negative human studies suffer from small cohort sizes, are retrospective and/or nonrandomized studies, and lack early clinical outcome as endpoint. Nevertheless, most, but not all, studies demonstrated improved functional parameters of the left ventricle, such as ejection fraction as measured by echocardiogram. Several ongoing multicenter trials on pharmacological cardioprotection, such as OVERCOME [82], PRADA [87], and/or MANTICORE- 101 breast [88] will provide more insight into the effects of combined treatment with beta-blockers and ACE inhibitors on anthracycline-induced cardiotoxicity or in the setting of trastuzumab therapy. There are novel options for cardioprotective therapies such as stem cell therapy, reviewed above that are underway for the investigation. Accordingly, a number of stem cell approaches for 
preservation or expansion of the cardiac progenitor cell pool have been investigated in animal models of anthracycline-induced cardiomyopathy. Although these alternative therapies are not ready yet for the phase of clinical translation, they may change the onset and development of cardiovascular damage by cytotoxic drugs. Pharmacological strategies as well as stem cell preparations are promising options for cardioprotection; however, results are still controversial and the stem cell strategies did not reach the clinical translation phase. With regard to human studies, the reasons for some negative results with betablockers and ACE inhibitors can be attributed to small patient cohorts and poorly designed clinical studies. With respect to stem cell-mediated cardioprotection, unfortunately human studies have not been designed so far that could reveal the relevance of the cardiac stem cell compartment in the pathophysiology of cancer therapy-induced cardiotoxicity, and the role of stem cell therapies aimed at the preservation or expansion of the cardiac progenitor cell pool.

\section{Recommendations}

In this SIC WG Position Paper, we have outlined basic mechanisms of cardiac toxicity induced by antineoplastic drugs and discussed promising therapeutic strategies for cardioprotection. We would like to make the following recommendations when assessing in preclinical models the clinical potential of a novel cardioprotective therapy: (1) The experimental models of cancer therapy-induced cardiotoxicity must include confounding factors such as comorbidities (such as age, diabetes, hypertension, dyslipidemia) and concomitant medication, which are known to potentially have an impact on the extend of antineoplastic drug-induced cardiotoxicity; (2) in the preclinical assessment, the novel cardioprotective therapy should be demonstrated to be effective after taking into account the effect of the above-mentioned confounding factors, known to potentially have an impact on cardioprotection.

\section{Conclusion}

Pharmacological and nonpharmacological attempts that aim to protect the heart against cardiotoxicity in cancer patients are encouraging, but human level data are presently not enough to support the effects of these interventions. Furthermore, there are several limitations of the preclinical models of cardiotoxicity that could hamper direct translation of new findings, including co-treatments, risk factors for cardiovascular disease, and other co-morbidities. In this SIC WG Position Paper, we share novel insights and potential targets that can be used for cardioprotection, providing the potential rationale for both existing and novel therapy, and noting areas where further research is needed. We also provide recommendations for optimizing the process of clinical translation of novel cardioprotective therapies tested in preclinical models of antineoplastic drug-induced cardiotoxicity. In regard to mechanisms and targets for cardioprotection, a central role is still played by the generation of ROS in the cardiomyocytes, which is considered as criticalmediator ofmyocardial damage during cancer therapy, especially anthracyclinemediated cardiotoxicity. Additional pathways including NRG1/ErbB pathway and tyrosine kinase receptors should be considered as responsible for the deterioration of cardiac performance as well. Alternatively, or rather additionally, a novel mechanism explaining antineoplastic drug-induced cardiotoxicity is given by depletion and/or dysfunction of the CSC compartment, which in turn might expire a more complex approach to the cardioprotection. Here, cardioprotective effects can be achieved via growth factors secreted by CSCS/CPCs, which can therefore influence several levels of protection in the myocardium in the course of antineoplastic drug-induced cardiotoxicity. The translation of novel cardioprotective approaches into the clinical setting for cancer patients has been challenging for the above-mentioned shortcomings of preclinical models and for the absence of clinical trials adequately designed and sufficiently empowered. 
Therefore, large clinical studies and more complex preclinical models will be necessary to get more insight into the clinical applicability of the novel cardioprotective strategies.

\section{Compliance with Ethical Standards}

Conflict of interest The authors declare that there are no conflicts of interest. 


\section{References}

1. Swain SM, Whaley FS, Ewer MS (2003) Congestive heart failure in patients treated with doxorubicin: a retrospective analysis of three trials. Cancer 97:2869-2879

2. Pinder MC, Duan Z, Goodwin JS, Hortobagyi GN, Giordano SH (2007) Congestive heart failure in older women treated with adjuvant anthracycline chemotherapy for breast cancer. J Clin Oncol 25:3808-3815

3. Ewer SM, Ewer MS (2008) Cardiotoxicity profile of trastuzumab. Drug Saf 31:459-467

4. Sawyer DB, Zuppinger C, Miller TA, Eppenberger HM, Suter TM (2002) Modulation of anthracyclineinduced myofibrillar disarray in rat ventricular myocytes by neuregulin-1beta and anti-erbB2: potential mechanism for trastuzumab-induced cardiotoxicity. Circulation 105:1551-1554

5. Outomuro D, Grana DR, Azzato F, Milei J (2007) Adriamycininduced myocardial toxicity: new solutions for an old problem? Int J Cardiol 117:6-15

6. Wouters KA, Kremer LC, Miller TL, Herman EH, Lipshultz SE (2005) Protecting against anthracyclineinduced myocardial damage: a review of the most promising strategies. $\mathrm{Br} J$ Haematol 131:561-578

7. Olson RD, Mushlin PS (1990) Doxorubicin cardiotoxicity: analysis of prevailing hypotheses. FASEB J 4:3076-3086

8. Minotti G, Menna P, Salvatorelli E, Cairo G, Gianni L (2004) Anthracyclines: molecular advances and pharmacologic developments in antitumor activity and cardiotoxicity. Pharmacol Rev 56:185-229

9. Lyu YL, Kerrigan JE, Lin CP, Azarova AM, Tsai YC, Ban Y et al (2007) Topoisomerase llbeta mediated DNA double-strand breaks: implications in doxorubicin cardiotoxicity and prevention by dexrazoxane. Cancer Res 67:8839-8846

10. Zhang S, Liu X, Bawa-Khalfe T, Lu LS, Lyu YL, Liu LF et al (2012) Identification of the molecular basis of doxorubicin-induced cardiotoxicity. Nat Med 18:1639-1642

11. Pointon AV, Walker TM, Phillips KM, Luo J, Riley J, Zhang SD et al (2010) Doxorubicin in vivo rapidly alters expression and translation of myocardial electron transport chain genes, leads to ATP loss and caspase 3 activation. PLoS One 5:e12733

12. Gonzalvez F, Gottlieb E (2007) Cardiolipin: setting the beat of apoptosis. Apoptosis 12:877-885

13. Fisher PW, Salloum F, Das A, Hyder H, Kukreja RC (2005) Phosphodiesterase-5 inhibition with sildenafil attenuates cardiomyocyte apoptosis and left ventricular dysfunction in a chronic model of doxorubicin cardiotoxicity. Circulation 111:1601-1610

14. Di X, Gennings C, Bear HD, Graham L, Sheth CM, White KL Jr et al (2010) Influence of the phosphodiesterase- 5 inhibitor, sildenafil, on sensitivity to chemotherapy in breast tumor cells. Breast Cancer Res Treat 124:349-360

15. Penna C, Mancardi D, Rastaldo R, Losano G, Pagliaro P (2007) Intermittent activation of bradykinin B2 receptors and mitochondrial KATP channels trigger cardiac postconditioning through redox signaling. Cardiovasc Res 75:168-177

16. Pentassuglia L, Sawyer DB (2009) The role of neuregulin-1 beta/erbb signaling in the heart. Exp Cell Res 315:627-637

17. Hynes NE, Lane HA (2005) ERBB receptors and cancer: the complexity of targeted inhibitors. Nat Rev Cancer 5:341-354

18. Slamon DJ, Clark GM, Wong SG, Levin WJ, Ullrich A, McGuire WL (1987) Human breast cancer: correlation of relapse and survival with amplification of the HER-2/neu oncogene. Science 235:177-182 
19. Slamon DJ, Leyland-Jones B, Shak S, Fuchs H, Paton V, Bajamonde A et al (2001) Use of chemotherapy plus a monoclonal antibody against HER2 for metastatic breast cancer that overexpresses HER2. N Engl J Med 344:783-792

20. Suter TM, Ewer MS (2013) Cancer drugs and the heart: importance and management. Eur Heart J 34:1102-1111

21. Ewer MS, Ewer SM (2010) Troponin I provides insight into cardiotoxicity and the anthracyclinetrastuzumab interaction. J Clin Oncol 28:3901-3904

22. Odiete O, Hill MF, Sawyer DB (2012) Neuregulin in cardiovascular development and disease. Circ Res 111:1376-1385

23. Ky B, Vejpongsa P, Yeh ET, Force T, Moslehi JJ (2013) Emerging paradigms in cardiomyopathies associated with cancer therapies. Circ Res 113:754-764

24. Bersell K, Arab S, Haring B, Kuhn B (2009) Neuregulin-1/erbb4 signaling induces cardiomyocyte proliferation and repair of heart injury. Cell 138:257-270

25. Force T, Krause DS, Van Etten RA (2007) Molecular mechanisms of cardiotoxicity of tyrosine kinase inhibition. Nat Rev Cancer 7:332-344

26. De Keulenaer GW, Doggen K, Lemmens K (2010) The vulnerability of the heart as a pluricellular paracrine organ: lessons from unexpected triggers of heart failure in targeted ErbB2 anticancer therapy. Circ Res 106:35-46

27. Tocchetti CG, Ragone G, Coppola C, Rea D, Piscopo G, Scala S et al (2012) Detection, monitoring, and management of trastuzumab- induced left ventricular dysfunction: an actual challenge. Eur J Heart Fail $14: 130-137$

28. Eschenhagen T, Force T, Ewer MS, de Keulenaer GW, Suter TM, Anker SD et al (2012) Cardiovascular side effects of cancer therapies: a position statement from the Heart Failure Association of the European Society of Cardiology. Eur J Heart Fail 13:1-10

29. Crone SA, Zhao YY, Fan L, Gu Y, Minamisawa S, Liu Y et al (2002) ErbB2 is essential in the prevention of dilated cardiomyopathy. Nat Med 8:459-465

30. Ewer MS, Vooletich MT, Durand JB, Woods ML, Davis JR, Valero V et al (2005) Reversibility of trastuzumab-related cardiotoxicity: new insights based on clinical course and response to medical treatment. J Clin Oncol 23:7820-7826

31. Timolati F, Ott D, Pentassuglia L, Giraud MN, Perriard JC, Suter TM et al (2006) Neuregulin-1 beta attenuates doxorubicininduced alterations of excitation-contraction coupling and reduces oxidative stress in adult rat cardiomyocytes. J Mol Cell Cardiol 41:845-854

32. Schmidinger M, Zielinski CC, VogI UM, Bojic A, Bojic M, Schukro C et al (2008) Cardiac toxicity of sunitinib and sorafenib in patients with metastatic renal cell carcinoma. J Clin Oncol 26:5204-5212

33. Welti J, Loges S, Dimmeler S, Carmeliet P (2013) Recent molecular discoveries in angiogenesis and antiangiogenic therapies in cancer. J Clin Invest 123:3190-3200

34. Cheng H, Force $\mathrm{T}$ (2010) Molecular mechanisms of cardiovascular toxicity of targeted cancer therapeutics. Circ Res 106:21-34

35. Tocchetti CG, Gallucci G, Coppola C, Piscopo G, Cipresso C, Maurea C et al (2013) The emerging issue of cardiac dysfunction induced by antineoplastic angiogenesis inhibitors. Eur J Heart Fail 15:482-489

36. Folkman J (1971) Tumor angiogenesis: therapeutic implications. N Engl J Med 285:1182-1186 
37. Curigliano G, Cardinale D, Suter T, Plataniotis G, de Azambuja E, Sandri MT et al (2012) Cardiovascular toxicity induced by chemotherapy, targeted agents and radiotherapy: ESMO clinical practice guidelines. Ann Oncol 23(Suppl 7):vii155-vii166

38. Marone G, Granata F (2014) Angiogenesis, lymphangiogenesis and clinical implications. Preface. Chem Immunol Allergy 99: XI-XII

39. Hurwitz H, Fehrenbacher L, Novotny W, Cartwright T, Hainsworth J, Heim W et al (2004) Bevacizumab plus irinotecan, fluorouracil, and leucovorin for metastatic colorectal cancer. N Engl J Med 350:2335-2342

40. Miller KD, Chap LI, Holmes FA, Cobleigh MA, Marcom PK, Fehrenbacher L et al (2005) Randomized phase III trial of capecitabine compared with bevacizumab plus capecitabine in patients with previously treated metastatic breast cancer. J Clin Oncol 23:792-799

41. Gresset $S$, Westermeier $P$, Rademacher $S$, Ouzunova M, Presterl T, Westhoff $P$ et al (2010) Stable carbon isotope discrimination is under genetic control in the $\mathrm{C} 4$ species maize with several genomic regions influencing trait expression. Plant Physiol 164:131-143

42. Chintalgattu V, Ai D, Langley RR, Zhang J, Bankson JA, Shih TL et al (2010) Cardiomyocyte PDGFR-beta signaling is an essential component of the mouse cardiac response to load-induced stress. J Clin Invest 120:472-484

43. Chu TF, Rupnick MA, Kerkela R, Dallabrida SM, Zurakowski D, Nguyen L et al (2007) Cardiotoxicity associated with tyrosine kinase inhibitor sunitinib. Lancet 370:2011-2019

44. Khakoo AY, Kassiotis CM, Tannir N, Plana JC, Halushka M, Bickford C et al (2008) Heart failure associated with sunitinib malate: a multitargeted receptor tyrosine kinase inhibitor. Cancer 112:2500-2508

45. Motzer RJ, Hutson TE, Tomczak P, Michaelson MD, Bukowski RM, Rixe $O$ et al (2007) Sunitinib versus interferon alfa in metastatic renal-cell carcinoma. N Engl J Med 356:115-124

46. Telli ML, Witteles RM, Fisher GA, Srinivas S (2008) Cardiotoxicity associated with the cancer therapeutic agent sunitinib malate. Ann Oncol 19:1613-1618

47. Hasinoff BB, Patel D (2010) The lack of target specificity of small molecule anticancer kinase inhibitors is correlated with their ability to damage myocytes in vitro. Toxicol Appl Pharmacol 249:132-139

48. Anisimov A, Alitalo A, Korpisalo P, Soronen J, Kaijalainen S, Leppanen VM et al (2009) Activated forms of VEGF-C and VEGF-D provide improved vascular function in skeletal muscle. Circ Res 104:1302-1312

49. Loges S, Roncal C, Carmeliet P (2009) Development of targeted angiogenic medicine. J Thromb Haemost 7:21-33

50. Izumiya Y, Shiojima I, Sato K, Sawyer DB, Colucci WS, Walsh K (2006) Vascular endothelial growth factor blockade promotes the transition from compensatory cardiac hypertrophy to failure in response to pressure overload. Hypertension 47:887-893

51. Levy BI (2006) Microvascular plasticity and experimental heart failure. Hypertension 47:827-829

52. De Boer RA, Pinto YM, Van Veldhuisen DJ (2003) The imbalance between oxygen demand and supply as a potential mechanism in the pathophysiology of heart failure: the role of microvascular growth and abnormalities. Microcirculation 10: 113-126

53. Kerkela R, Woulfe KC, Durand JB, Vagnozzi R, Kramer D, Chu TF et al (2009) Sunitinib-induced cardiotoxicity is mediated by off-target inhibition of AMP-activated protein kinase. Clin Transl Sci 2:15-25

54. Bergmann O, Bhardwaj RD, Bernard S, Zdunek S, Barnabe- Heider F, Walsh S et al (2009) Evidence for cardiomyocyte renewal in humans. Science 324:98-102 
55. Bearzi C, Rota M, Hosoda T, Tillmanns J, Nascimbene A, De Angelis A et al (2007) Human cardiac stem cells. Proc Natl Acad Sci USA 104:14068-14073

56. De Angelis A, Piegari E, Cappetta D, Marino L, Filippelli A, Berrino L et al (2010) Anthracycline cardiomyopathy is mediated by depletion of the cardiac stem cell pool and is rescued by restoration of progenitor cell function. Circulation 121:276-292

57. Fazel S, Cimini M, Chen L, Li S, Angoulvant D, Fedak P et al (2006) Cardioprotective c-kit? cells are from the bone marrow and regulate the myocardial balance of angiogenic cytokines. J Clin Invest 116:1865-1877

58. Ladas EJ, Jacobson JS, Kennedy DD, Teel K, Fleischauer A, Kelly KM (2004) Antioxidants and cancer therapy: a systematic review. J Clin Oncol 22:517-528

59. van Dalen EC, Caron HN, Dickinson HO, Kremer LC (2008) Cardioprotective interventions for cancer patients receiving anthracyclines. Cochrane Database Syst Rev (2):CD003917.

doi:10.1002/14651858.CD003917.pub3

60. Huelsenbeck J, Henninger C, Schad A, Lackner KJ, Kaina B, Fritz G (2011) Inhibition of Rac1 signaling by lovastatin protects against anthracycline-induced cardiac toxicity. Cell Death Dis 2:e190

61. Riad A, Bien S, Westermann D, Becher PM, Loya K, Landmesser U et al (2009) Pretreatment with statin attenuates the cardiotoxicity of doxorubicin in mice. Cancer Res 69:695-699

62. Seicean S, Seicean A, Plana JC, Budd GT, Marwick TH (2012) Effect of statin therapy on the risk for incident heart failure in patients with breast cancer receiving anthracycline chemotherapy: an observational clinical cohort study. J Am Coll Cardiol 60:2384-2390

63. Acar Z, Kale A, Turgut M, Demircan S, Durna K, Demir S et al (2011) Efficiency of atorvastatin in the protection of anthracycline- induced cardiomyopathy. J Am Coll Cardiol 58:988-989

64. Scott JM, Khakoo A, Mackey JR, Haykowsky MJ, Douglas PS, Jones LW (2011) Modulation of anthracycline-induced cardiotoxicity by aerobic exercise in breast cancer: current evidence and underlying mechanisms. Circulation 124:642-650

65. Jones LW, Eves ND, Haykowsky M, Freedland SJ, Mackey JR (2009) Exercise intolerance in cancer and the role of exercise therapy to reverse dysfunction. Lancet Oncol 10:598-605

66. Weijl NI, Elsendoorn TJ, Lentjes EG, Hopman GD, Wipkink- Bakker A, Zwinderman AH et al (2004) Supplementation with antioxidant micronutrients and chemotherapy-induced toxicity in cancer patients treated with cisplatin-based chemotherapy: a randomised, double-blind, placebo-controlled study. Eur J Cancer 40:1713-1723

67. Giovannucci E, Chan AT (2010) Role of vitamin and mineral supplementation and aspirin use in cancer survivors. J Clin Oncol 28:4081-4085

68. Hardy ML (2008) Dietary supplement use in cancer care: help or harm. Hematol Oncol Clin N Am 22:581-617

69. Cardinale D, Colombo A, Lamantia G, Colombo N, Civelli M, De Giacomi G et al (2010) Anthracyclineinduced cardiomyopathy: clinical relevance and response to pharmacologic therapy. J Am Coll Cardiol 55:213-220

70. Okumura K, Jin D, Takai S, MiyazakiM(2002) Beneficial effects of angiotensin-converting enzyme inhibition in adriamycin-induced cardiomyopathy in hamsters. Jpn J Pharmacol 88:183-188

71. Tokudome T, Mizushige K, Noma T, Manabe K, Murakami K, Tsuji T et al (2000) Prevention of doxorubicin (adriamycin)- induced cardiomyopathy by simultaneous administration of angiotensinconverting enzyme inhibitor assessed by acoustic densitometry. J Cardiovasc Pharmacol 36:361-368 
72. Lopez-Sendon J, Swedberg K, McMurray J, Tamargo J, Maggioni AP, Dargie H et al (2004) Expert consensus document on angiotensin converting enzyme inhibitors in cardiovascular disease. The task force on ACE-inhibitors of the European Society of Cardiology. Eur Heart J 25:1454-1470

73. Cernecka H, Ochodnicka-Mackovicova K, Kucerova D, Kmecova J, Nemcekova V, Doka G et al (2013) Enalaprilat increases PPARbeta/delta expression, without influence on PPARalpha and PPARgamma, and modulate cardiac function in sub-acute model of daunorubicin-induced cardiomyopathy. Eur J Pharmacol 714:472-477

74. Soga M, Kamal FA, Watanabe K, Ma M, Palaniyandi S, Prakash P et al (2006) Effects of angiotensin II receptor blocker (candesartan) in daunorubicin-induced cardiomyopathic rats. Int J Cardiol 110:378-385

75. Nakamae H, Tsumura K, Terada Y, Nakane T, Nakamae M, Ohta K et al (2005) Notable effects of angiotensin II receptor blocker, valsartan, on acute cardiotoxic changes after standard chemotherapy with cyclophosphamide, doxorubicin, vincristine, and prednisolone. Cancer 104:2492-2498

76. Shi Y, Moon M, Dawood S, McManus B, Liu PP (2011) Mechanisms and management of doxorubicin cardiotoxicity. Herz 36:296-305

77. Bovelli D, Plataniotis G, Roila F (2010) Cardiotoxicity of chemotherapeutic agents and radiotherapyrelated heart disease: ESMO clinical practice guidelines. Ann Oncol 21(Suppl 5): v277-v282

78. Seicean S, Seicean A, Alan N, Plana JC, Budd GT, Marwick TH (2013) Cardioprotective effect of betaadrenoceptor blockade in patients with breast cancer undergoing chemotherapy: follow-up study of heart failure. Circ Heart Fail 6:420-426

79. Kaya MG, Ozkan M, Gunebakmaz O, Akkaya H, Kaya EG, Akpek M et al (2013) Protective effects of nebivolol against anthracycline-induced cardiomyopathy: a randomized control study. Int J Cardiol 167:2306-2310

80. Oliva S, Cioffi G, Frattini S, Simoncini EL, Faggiano P, Boccardi L et al (2012) Administration of angiotensin-converting enzyme inhibitors and beta-blockers during adjuvant trastuzumab chemotherapy for nonmetastatic breast cancer: marker of risk or cardioprotection in the real world? Oncologist 17:917924

81. Kalay N, Basar E, Ozdogru I, Er O, Cetinkaya Y, Dogan A et al (2006) Protective effects of carvedilol against anthracyclineinduced cardiomyopathy. J Am Coll Cardiol 48:2258-2262

82. Bosch X, Rovira M, Sitges M, Domenech A, Ortiz-Perez JT, de Caralt TM et al (2013) Enalapril and carvedilol for preventing chemotherapy-induced left ventricular systolic dysfunction in patients with malignant hemopathies: the OVERCOME trial (preventiOn of left Ventricular dysfunction with Enalapril and caRvedilol in patients submitted to intensive ChemOtherapy for the treatment of Malignant hEmopathies). J Am Coll Cardiol 61:2355-2362

83. Georgakopoulos P, Roussou P, Matsakas E, Karavidas A, Anagnostopoulos N, Marinakis T et al (2012) Cardioprotective effect of metoprolol and enalapril in doxorubicin-treated lymphoma patients: a prospective, parallel-group, randomized, controlled study with 36-month follow-up. Cardiology 123:240247

84. Kim IM, Tilley DG, Chen J, Salazar NC, Whalen EJ, Violin JD et al (2008) Beta-blockers alprenolol and carvedilol stimulate beta-arrestin-mediated EGFR transactivation. Proc Natl Acad Sci USA 105:14555-14560 85. Zhang X, Szeto C, Gao E, Tang M, Jin J, Fu Q et al (2013) Cardiotoxic and cardioprotective features of chronic betaadrenergic signaling. Circ Res 112:498-509

86. Nakamura K, Kusano K, Nakamura Y, Kakishita M, Ohta K, Nagase S et al (2002) Carvedilol decreases elevated oxidative stress in human failing myocardium. Circulation 105:2867-2871 
87. Heck SL, Gulati G, Ree AH, Schulz-Menger J, Gravdehaug B, Rosjo H et al (2012) Rationale and design of the prevention of cardiac dysfunction during an adjuvant breast cancer therapy (PRADA) trial. Cardiology

123:240-247

88. Pituskin E, Haykowsky M, Mackey JR, Thompson RB, Ezekowitz J, Koshman S et al (2011) Rationale and design of the multidisciplinary approach to novel therapies in cardiology oncology research trial

(MANTICORE 101-breast): a randomized, placebo-controlled trial to determine if conventional heart failure pharmacotherapy can prevent trastuzumab-mediated left ventricular remodeling among patients with HER2? early breast cancer using cardiac MRI. BMC Cancer 11:318

89. Oliveira MS, Melo MB, Carvalho JL, Melo IM, Lavor MS, Gomes DA et al (2013) Doxorubicin cardiotoxicity and cardiac function improvement after stem cell therapy diagnosed by strain echocardiography. J Cancer Sci Ther 5:52-57

90. Di GH, Jiang S, Li FQ, Sun JZ, Wu CT, Hu X et al (2012) Human umbilical cord mesenchymal stromal cells mitigate chemotherapy-associated tissue injury in a pre-clinical mouse model. Cytotherapy 14:412-422

91. Merino H, Singla DK (2014) Notch-1 mediated cardiac protection following embryonic and induced pluripotent stem cell transplantation in doxorubicin-induced heart failure. PLoS One 9:e101024

92. Singla DK (2014) Akt-mTOR pathway inhibits apoptosis and fibrosis in doxorubicin-induced cardiotoxicity following embryonic stem cell transplantation. Cell Transplant 24(6):1031-1042

93. Singla DK, Abdelli LS (2014) Embryonic stem cells and released factors stimulate c-kit/FLK-1 progenitor cells and promote neovascularization in doxorubicin-induced cardiomyopathy. Cell Transplant 24(6):10431052

94. Madonna R, Rokosh G, De Caterina R, Bolli R (2010) Hepatocyte growth factor/Met gene transfer in cardiac stem cells - potential for cardiac repair. Basic Res Cardiol 105:443-452

95. Herman EH, Ferrans VJ (1998) Preclinical animal models of cardiac protection from anthracyclineinduced cardiotoxicity. Semin Oncol 25:15-21

96. Lipshultz SE, Cohen H, Colan SD, Herman EH (2006) The relevance of information generated by in vitro experimental models to clinical doxorubicin cardiotoxicity. Leuk Lymphoma 47:1454-1458

97. Zbinden G, Bachmann E, Holderegger C (1971) Model systems for cardiotoxic effects of anthracyclines. Antibiot Chemother 1978(23):255-270

98. Cardinale D, Colombo A, Sandri MT, Lamantia G, Colombo N, Civelli M et al (2006) Prevention of highdose chemotherapyinduced cardiotoxicity in high-risk patients by angiotensinconverting enzyme inhibition. Circulation 114:2474-2481

99. Piegari E, Di Salvo G, Castaldi B, Vitelli MR, Rodolico G, Golino P et al (2008) Myocardial strain analysis in a doxorubicin- induced cardiomyopathy model. Ultrasound Med Biol 34:370-378

100. Adamcova M, Simunek T, Kaiserova H, Popelova O, Sterba M, Potacova A et al (2007) In vitro and in vivo examination of cardiac troponins as biochemical markers of drug-induced cardiotoxicity. Toxicology 237:218-228

101. Madonna R, Delli Pizzi S, Di Donato L, Mariotti A, Di Carlo L, D’Ugo E et al (2012) Non-invasive in vivo detection of peripheral limb ischemia improvement in the rat after adipose tissue-derived stromal cell transplantation. Circ J 76:1517-1525

102. Madonna R, Delli Pizzi S, Tartaro A, De Caterina R (2014) Transplantation of mesenchymal cells improves peripheral limb ischemia in diabetic rats. Mol Biotechnol 56:438-448 
Table 1 Novel cardioprotective strategies

Table 1 Novel candioprotective strategies

\begin{tabular}{|c|c|c|}
\hline Treatment & Mechanism & Results \\
\hline Probucol & ROS reduction & No benefits \\
\hline Vitamin E & ROS reduction & No benefits \\
\hline $\begin{array}{l}N \text { - } \\
\text { acetyleysteine }\end{array}$ & ROS reduction & No benefits \\
\hline Dexracoxane & $\begin{array}{l}\text { ROS reduction } \\
\text { Reduction in DNA } \\
\text { damage }\end{array}$ & $\mathbf{H F} \downarrow$ \\
\hline Statin & $\begin{array}{l}\text { Reduction in DNA } \\
\text { damage } \\
\text { Reduction in cardiac } \\
\text { fibrosis }\end{array}$ & $\mathbf{H F} \perp$ \\
\hline ACE inhibitors & $\begin{array}{l}\text { ROS reduction } \\
\text { Reduction in cardiac } \\
\text { fibrosis } \\
\text { Downregulation of } \\
\text { NRG - IErbB pathway }\end{array}$ & $\mathbf{H F} \perp$ \\
\hline Beta-blockers & $\begin{array}{l}\text { Downregulation of } \\
\text { catecholamine } \\
\text { stimulation } \\
\text { Enhanced lusitropy }\end{array}$ & HF 1 \\
\hline Stem cells & $\begin{array}{l}\text { Paracrine effects with } \\
\text { reduction in cardiac } \\
\text { fibrosis, } \\
\text { cardioprotection }\end{array}$ & $\begin{array}{l}\text { Improved heart function } \\
\text { in preclinical models } \\
\text { of doxorubicin- } \\
\text { induced } \\
\text { candiomyopathy }\end{array}$ \\
\hline $\begin{array}{l}\text { Aerobic } \\
\text { exercise }\end{array}$ & ROS reduction & $\begin{array}{l}\text { No benefits in } \\
\text { trastuzumab-treated } \\
\text { patients }\end{array}$ \\
\hline & & $\begin{array}{l}\text { Reduction in all-cause } \\
\text { mortality in } \\
\text { anthracycline-treated } \\
\text { patients }\end{array}$ \\
\hline Nutraceuticals & ROS reduction & $\begin{array}{l}\text { Reduction in } \\
\text { chemotherapy } \\
\text { collateral effects } \\
\text { No proven } \\
\text { cardioprotection }\end{array}$ \\
\hline
\end{tabular}

HF heart failure, ACE angiotensin-converting enzyme, ROS reactive oxygen species 